\title{
L'Estonie : un nouveau modèle éducatif
}

\section{Stéphane Kesler}

\section{OpenEdition}

\section{Journals}

Édition électronique

URL : https://journals.openedition.org/ries/5880

DOI : 10.4000/ries.5880

ISSN : 2261-4265

\section{Éditeur}

France Education international

\section{Édition imprimée}

Date de publication : 1 septembre 2017

Pagination : 19-22

ISBN : 978-2-85420-615-9

ISSN : 1254-4590

\section{Référence électronique}

Stéphane Kesler, "L'Estonie : un nouveau modèle éducatif », Revue internationale d'éducation de Sèvres [En ligne], 75 | septembre 2017, mis en ligne le 01 septembre 2019, consulté le 24 juin 2021. URL : http://journals.openedition.org/ries/5880 ; DOI : https://doi.org/10.4000/ries.5880

Ce document a été généré automatiquement le 24 juin 2021.

(c) Tous droits réservés 


\title{
L'Estonie : un nouveau modèle éducatif
}

\author{
Stéphane Kesler
}

1 Avec 1,3 million d'habitants, l'Estonie est un pays faiblement peuplé. Sa population scolaire compte 143000 élèves dans les premier et second degrés. Pourtant, cette taille réduite n'empêche pas l'Estonie non seulement de disposer d'un système éducatif original, mais aussi de représenter un modèle de réussite scolaire. La position de l'Estonie dans l'évaluation PISA de 2012 était déjà très favorable, mais les résultats de 2015 sont spectaculaires : l'Estonie a dépassé la Finlande et figure désormais au premier rang européen du classement PISA et au troisième rang mondial. Cette réussite ne doit rien au hasard: il existe un modèle éducatif estonien, qui se caractérise par quelques éléments originaux.

\section{Une volonté d'excellence éducative globale inscrite dans une perspective internationale}

2 L'Estonie dispose d'une tradition ancienne de qualité scolaire. Selon un recensement effectué en 1881, $48 \%$ de la population savaient déjà lire et $94 \%$ lire et écrire, bien audessus de la plupart des pays européens. Elle a poursuivi cette priorité donnée à l'éducation: en 2011, la part de la population comprise entre 55 et 64 ans ayant été scolarisée au-delà du collège était de $85 \%$ en Estonie, soit 25 points au-dessus de la moyenne française.

3 L'excellence éducative recherchée est globale et ne s'arrête pas aux portes de l'école. Considérant chaque individu comme devant être en situation permanente d'apprentissage, les objectifs fixés au système éducatif doivent se traduire dans les compétences de l'ensemble de la population, et un certain nombre d'indicateurs clés de la stratégie 2020 du ministère de l'éducation concernent les adultes.

Cette volonté d'excellence s'intègre aussi dans une dimension internationale. Membre de l'OCDE depuis 2010, l'Estonie participe à PISA depuis 2006, ainsi qu'aux principaux 
tests internationaux (dont TALIS et PIAAC). Au-delà, les politiques éducatives se réfèrent explicitement à l'environnement international. À titre d'exemple, parmi les indicateurs clés de la stratégie éducative élaborée en 2014, figure l'accroissement du nombre d'élèves les plus performants identifiés dans PISA. La réussite dans les évaluations internationales permet en retour de valider, dans le débat public estonien, les choix éducatifs réalisés depuis une génération.

\section{Un système éducatif fondé sur l'information}

L'Estonie a fait le choix de participer pleinement à la société de l'information, et l'État contribue à ce projet de société digitale, en offrant le plus de services dématérialisés possible, qu'il s'agisse du vote électronique ou de la possibilité de signer en ligne des contrats authentifiés.

En termes éducatifs, ce primat de l'information se décline de deux façons.

7 D'une part, les apprentissages font une large part à l'excellence numérique. L'inscription de l'éducation dans la société de l'information est l'un des cinq objectifs de la stratégie éducative 2020 de l'Estonie. Parmi les indicateurs choisis figure la maitrise par chaque élève des technologies de l'information et de la communication, et le fait que $80 \%$ des adultes (compris entre 16 et 74 ans) disposent de compétences en la matière.

D'autre part, l'Estonie a développé un système d'information exhaustif qui est au cœur du pilotage du système éducatif : l'Estonian Education Information System (EHIS). Son champ couvre l'ensemble du champ éducatif, depuis l'enseignement préélémentaire jusqu'au supérieur et à la formation d'adultes. Il comprend les principaux domaines concernés par l'éducation: les évaluations d'élèves; le management des écoles; les curricula; les manuels scolaires; la recherche en éducation; l'entrée dans l'enseignement supérieur; le registre des qualifications professionnelles. Il est consultable par tous les publics directement parties prenantes de l'école: élèves et étudiants, enseignants, institutions éducatives.

L'intérêt de ce système unique est de pouvoir disposer d'informations et de statistiques portant sur l'intégralité du champ éducatif, et ce à toutes les échelles : d'établissement, locale, nationale, internationale (UNESCO, OCDE, Eurostat).

Le croisement de ces informations conduit à des comparaisons temporelles ou géographiques entre établissements ou régions, en facilite le pilotage, et permet de passer de la description de l'activité à l'analyse des résultats de l'enseignement, en établissant un lien entre les différentes échelles de l'action éducative.

\section{Une autonomie de l'établissement qui intègre l'offre d'enseignement}

11 En Estonie, l'État central dispose peu de pouvoir en matière éducative. Symboliquement, le siège du ministère de l'éducation se situe à Tartu, ancienne ville universitaire, et non à Tallin, la capitale politique et le siège de toutes les autres institutions. À l'instar des pays nordiques auxquels l'Estonie se compare, les pouvoirs de l'échelon local, municipalité et province, sont plus importants que ceux de l'État 
central et comprennent notamment la contractualisation avec les établissements scolaires et l'obtention de leurs moyens.

Toutefois, la particularité de l'Estonie est surtout d'accorder un très haut niveau d'autonomie à l'établissement scolaire. L'OCDE réalise une évaluation globale du degré d'autonomie, selon le pourcentage de décisions prises au niveau de l'établissement. Les compétences transférées aux établissements peuvent correspondre à trois domaines principaux: les ressources humaines (recrutement des enseignants et des chefs d'établissement); le financement (utilisation d'un budget public et capacité à faire appel à des ressources privées); l'enseignement et la pédagogie (choix du programme ou des matériels).

13 Dans l'ensemble, l'Estonie figure dans le trio de tête de l'OCDE, avec l'Angleterre et les Pays-Bas, puisque plus de $70 \%$ des décisions sont prises au niveau de l'établissement scolaire.

utre, l'autonomie de l'établissement tend à changer de nature. Elle ne concerne plus seulement son fonctionnement et sa capacité à utiliser les marges de manœuvre qui lui ont été attribuées, à l'occasion du mouvement général de décentralisation observé partout en Europe depuis une génération. Ces marges de manœuvre sont en effet le plus souvent limitées, parce que si les établissements disposent de davantage de compétences et d'une liberté d'action accrue, c'est presque toujours en contrepartie d'un contrôle renforcé par des collectivités locales à la fois plus attentives et plus puissantes.

Ici, l'autonomie est comprise dans une dimension plus ambitieuse: la capacité de l'établissement à proposer et à porter un projet éducatif singulier. L'établissement peut ainsi construire le cadre de son action et ses objectifs propres, ce qui détermine en conséquence ses relations avec ses tutelles et la grille selon laquelle il pourra être évalué. Le débat porte donc moins sur l'utilisation des moyens que sur la qualité des enseignements dispensés, en fonction des objectifs que se fixe l'établissement. C'est son ambition pédagogique qui est discutée, et non les facteurs qui permettent d'y parvenir.

Il ne s'agit pas de construire une éducation à la carte : le curriculum assez large va de pair avec un socle commun, des disciplines partagées et des examens terminaux (et partiels) dont les résultats sont regardés par tous avec attention. L'autonomie consiste plutôt à pouvoir déterminer une offre d'enseignement cohérente et propre à un établissement. En Estonie, un collège peut décider de proposer des cours conjointement avec des enseignants de lycée, sur des spécialités qu'il souhaite développer. Il ne s'agit plus d'enseignements optionnels mais d'un positionnement qui fonde la stratégie de l'établissement, sa différenciation avec d'autres, et qui se traduit, notamment, par des objectifs précis concernant les savoirs des élèves, évalués tout au long de l'année. L'autonomie de l'établissement consiste donc à être à l'origine de son cadre d'action.

\section{Un établissement acteur de son évaluation}

17 L'Estonie représente un modèle original d'évaluation d'établissement. Historiquement, les premiers dispositifs, développés en Angleterre par l'inspection anglaise, l'ofsted, à partir de la fin des années 1980, ont renforcé l'encadrement éducatif de l'école. Parallèlement à l'autonomie conférée aux établissements, une autorité extérieure structure l'inspection d'établissements: l'établissement est certes autonome dans 
l'utilisation de ses moyens mais il en rend compte à travers un schéma unique. L'évaluation d'établissement, "school inspection ", se fonde sur un cadre essentiellement chiffré et s'inscrit dans une démarche comparatiste proposée tant aux parents qu'aux autorités de tutelle.

Un nouveau modèle d'évaluation d'établissements, prenant davantage en compte leurs forces propres, s'est ensuite développé. Une place plus importante est faite aux équipes des établissements dans la mise en place de leur évaluation : l'approche est davantage collaborative, plus qualitative et tournée vers l'amélioration de l'établissement et non plus sur son classement. Porté d'abord par l'Écosse, ce modèle s'est diffusé largement en Europe et est davantage compatible avec l'autonomie croissante des établissements, mais il se réfère toujours à un cadre fixé à l'extérieur de l'établissement.

Face à ces modèles fondés sur le primat de l'évaluation externe, l'Estonie représente une nouvelle conception, qui prend en compte l'autonomie pédagogique de l'établissement et se fonde essentiellement sur son auto-évaluation. L'évaluation externe de l'ensemble des établissements, sur une grille commune, n'est plus réalisée à l'échelle d'un pays. De même, il n'y a plus d'inspection d'écoles, à intervalles réguliers, réalisée par un organisme dépendant d'autorités éducatives locales ou centrales. En revanche, l'établissement doit conduire chaque année sa propre évaluation. Libre à lui de la mener comme il l'entend: les méthodes comme les indicateurs retenus sont choisis par lui, en fonction de ses objectifs pédagogiques spécifiques. En revanche, celle-ci doit être cohérente avec sa stratégie et la qualité de l'auto-évaluation comme la véracité des données fournies sont des éléments déterminants de jugement de l'établissement par les autorités de tutelle: l'établissement est acteur de son évaluation, il en devient donc responsable.

Par ces quelques caractéristiques majeures, l'Estonie prend ainsi résolument place, quoique pour l'instant de manière discrète, parmi les systèmes éducatifs les plus performants au monde.

\section{INDEX}

Palabras claves : autonomía, instituciones de enseñanza, evaluación, información, sistema educativo

Keywords : autonomy, educational institutions, evaluation, information, educational system Index géographique : Estonie

Mots-clés : autonomie, établissement d'enseignement, évaluation, information, système éducatif 


\section{AUTEUR}

\section{STÉPHANE KESLER}

Stéphane Kesler est inspecteur général de l'administration de l'éducation nationale et de la recherche (France). Courriel : stephane.kesler@education.gouv.fr 